\title{
POLÍTICAS DE INOVAÇÃO PARA PAÍSES EM DESENVOLVIMENTO
}

João Batista Pamplona ${ }^{1}$

Ana Carolina Penha ${ }^{2}$

\section{RESUMO}

O objetivo do presente trabalho é revisar a literatura, a partir de uma abordagem neoschumpeteriana, sobre políticas que visem o desempenho das inovações nos países em desenvolvimento.

A inovação sempre desempenhou um papel importante no conjunto de ações voltadas ao desenvolvimento econômico e social dos países: ajuda a melhorar a produtividade, é a base da competitividade entre e intra países e melhora o bem-estar dos cidadãos. No atual contexto de "poli-crise", a inovação se faz imperativa (BANCO MUNDIAL, 2010). Além da tecnologia, a adaptação às alterações climáticas, o ajustamento dos limites dos recursos naturais, a proteção da biodiversidade e a inclusão social são pautas que requerem, fundamentalmente, novos padrões de produção e consumo que podem ser estabelecidos a partir das inovações, reguladas através de políticas.

Ter uma política de inovação é muito relevante porque vai além da política tradicional de ciência e tecnologia e envolve muitos

1 Professor do Programa de Pós-graduação em Economia Política da PUC-SP do Programa de Pós-graduação em Administração da USCS. pamplona@pucsp.br.

2 Mestranda do Programa de Pós-graduação em Economia Política da PUC-SP.carolina.penha@gmail.com. 
departamentos governamentais. Mais que coordenar as ações inovadoras presentes em diversos setores, a política de inovação propicia um ambiente favorável ao surgimento de novas inovações, além de ser um instrumento político eficiente para desencadear mudanças e melhorias nas condições-quadro gerais do país (OECD, 2012).

Palavras-chave: Política de Inovação, Inovação, Neoschumpeterianos, Países em Desenvolvimento

\section{INTRODUÇÃO}

Apontada como essencial para o processo de desenvolvimento econômico por Joseph Schumpeter (1883-1950), a inovação tem ganhado cada vez mais centralidade. Até meados da década de 1960, a inovação era tratada como um processo linear que compreendia uma sequência de estágios que se iniciava na pesquisa básica, passando à pesquisa aplicada, desenvolvimento, produção e resultava, ao fim da cadeia, na difusão de determinada ideia ou produto oriundos do avanço do conhecimento científico (science push) ou da própria demanda por novas tecnologias (demand pull) (CASSIOLATO; LASTRES, 2005).

O entendimento do processo de inovação passa a ser aprimorado nas décadas seguintes, principalmente devido a programas de pesquisas empíricas importantes que demonstraram que a inovação não é resultado de uma ação ou necessidade isolada, mas sim de um ambiente bem desenvolvido e bem estruturado institucionalmente e que representa processos de desenvolvimento não lineares, mas sim interativos. Um dos primeiros programas de pesquisa que contribuiu para esse novo olhar para a inovação foi o Projeto SAPPHO (Scientific Activity Predictor From Patterns With Heuristic Organs), coordenado por Chris Freeman da Universidade de Sussex. A pesquisa, apresentada em Freeman e Soete (2008), analisou 58 iniciativas de inovação, comparando as que haviam obtido sucesso com outras que não se concretizaram. Os resultados apontaram que a comunicação com fontes externas às firmas e uma boa comunicação com os usuários de tais inovações foram a chave para o sucesso dos inovadores. As inovações que não apresentavam tais características estavam fadadas ao fracasso.

É cada vez mais consenso entre os autores que para uma economia lograr sucesso na geração e difusão de inovações é necessário que haja colaboração, não apenas entre as empresas (redes de empresas), mas entre o Estado, as universidades e as firmas. 
Segundo a OCDE (2012), ter uma política de inovação é essencial em todas as fases do desenvolvimento econômico de um país, especificamente no momento de criação e difusão de tecnologias relevantes para o crescimento econômico. No entanto, a presença da inovação vai além da propagação de tecnologia (high-tech myopia). Por meio da inovação é possível enfrentar os desafios específicos do contexto socioeconômicos local, como por exemplo, a busca por uma sociedade mais igualitária (inclusividade), a otimização de ambientes de trabalho e a promoção de um meio ambiente cada vez mais sustentável.

Para tal, a inovação depende significativamente das condições gerais de governança, educação e infraestrutura da economia. Tais condições são normalmente consideradas como variáveis problemáticas nos países em desenvolvimento, no entanto, de acordo com o Banco Mundial (2010), a experiência mostra não só que políticas de inovação são possíveis e eficazes, mas também que elas ajudam a criar ambientes para reformas mais amplas. Em última análise, uma estratégia econômica bem-sucedida tem de construir amplas capacidades de inovação para fomentar o crescimento (OCDE, 2012).

O objetivo do presente artigo é apontar a importância e analisar que características essenciais a política de inovação deve ter para atender as necessidades específicas dos países em desenvolvimento na sua estratégia de catching up. Para isso, é feita revisão crítica da literatura neoschumpeteriana. $\mathrm{O}$ artigo se divide em três seções, além das considerações finais e desta introdução. A primeira seção da trata de apresentar a evolução do conceito de política de inovação. A segunda aborda a importância da política de inovação. A terceira seção analisa as características essenciais das políticas de inovação para o catching up.

\section{POLÍTICA DE INOVAÇÃO: UM CONCEITO}

A noção do que é política de inovação surgiu, em meados dos anos 1990, da necessidade de se expandir o conceito de políticas de ciência e tecnologia, que até então era vigente. Sendo assim, a reflexão teórica do que é a política de inovação e como ela pode ser analisada ainda é de certa forma recente (FARGERBERG, 2014).

Há um consenso na comunidade de pesquisadores da área de inovação de que há duas abordagens principais relacionadas à política de inovação: a abordagem ampla (broad approach) e a abordagem restrita (narrow approach) (MAKÓ; ILLÉSSY, 
2015). A escolha de qual abordagem se encaixará melhor para determinado país, de acordo com Fagerberg (2014), depende dos fundamentos teóricos que os próprios policymakers utilizam para o conceito de inovação. Em outras palavras, o processo de formulação de políticas de inovação depende de considerações teóricas.

As políticas de inovação com abordagem restrita (narrow approach) tratam exclusivamente das políticas que foram criadas com a intenção de impactar diretamente a inovação em determinado setor (MAKÓ; ILLÉSSY, 2015). Esta abordagem entende a inovação como resultado de atividades científicas consideradas como o principal fator causal do progresso econômico (Science push). É também conhecido como "modelo linear de inovação", que foi por muito tempo tido como modelo dominante para política de inovação. Nesse tipo de abordagem, todas as atividades de inovação começam com a pesquisa científica básica, cujo resultado é então transformado em engenharia e fabricação; e então o novo produto é vendido por meio de ações de marketing e vendas. Aqui, as formas não tecnológicas, tais como inovações organizacionais e de marketing são assumidas como de menor importância.

Além disso, neste tipo de abordagem, a inovação é fortemente ligada a algo radicalmente novo, seja produto ou processos. Inovações incrementais são vistos como de importância secundária. Fargerberg (2014) afirma que, na abordagem restrita, a política de inovação tem lugar principalmente no setor da indústria transformadora, muitas vezes considerado como a espinha dorsal da atividade econômica.

Makó e Illéssy (2015), em complemento à ideia do science push, destacam outro tipo de modelo linear de inovação conhecido por "modelo de demanda" (demand pull), na qual o gatilho dos processos de inovação não é a ciência, mas as necessidades do mercado. A lógica deste modelo é bastante semelhante ao modelo que prioriza a tecnologia e ciência, ou seja, as relações entre os elementos do processo de inovação são unívocas e quase não há interação com fontes externas.

Em contrapartida, a abordagem ampla (broad approach) considera todas as políticas ou instrumentos de política que influenciam a inovação ou o ambiente em que essa está inserida. Neste tipo de interpretação, a inovação é tratada como um processo contínuo relacionado à prática cotidiana da organização. É levado em conta não apenas a capacidade de desenvolvimento tecnocientífico, mas também as habilidades organizacionais, a identificação de oportunidades, o desenvolvimento e acumulação de competências diversas e abrangentes por parte das organizações, 
consideradas mais importantes do que as conquistas puramente técnicas. Também se salienta a importância de inovações incrementais e não apenas as inovações disruptivas.

A abordagem ampla de inovação implica uma estratégia de formulação de políticas diferente em comparação com o modelo linear ou modelo de abordagem restrita. Isto se dá, principalmente, porque o caráter interativo da inovação, aqui muito enfatizado, deve ser levado em conta. As relaçōes de confiança, a forte cooperação e as intensas interações sociais entre os atores envolvidos asseguram o fluxo de informação necessário e moldam continuamente os processos de aprendizagem desempenhando um papel central nesta abordagem (MAKÓ; ILLÉSSY, 2015). Esta abordagem mais ampla na formulação de políticas é mais bem refletida, por exemplo, pela corrente teórica dos Sistemas Nacionais de Inovação (SNI) ou do Modelo Tripla Hélice (Triple Helix).

O SNI, conceituado pela primeira vez por Lundvall (1988; 1992), foi desenvolvido como uma resposta teórica por parte dos evolucionistas/neoschumpeterianos ao modelo linear da inovação. $\mathrm{O}$ autor destaca a importância da relação entre as empresas e a universidade no fomento da inovação em diversas áreas da economia. Embora englobe as três esferas limitadas a uma fronteira nacional (a saber: Estado, iniciativa privada e academia), o SNI, como afirma Henry Etzkowitz e Loet Leydesdorff $(1995$; 2000), prioriza muito a relação entre as firmas e as universidades e como o fruto deste relacionamento pode servir de combustível à inovação. "Não é surpreendente que a ligação entre universidades e indústria se tenha tornado uma questão política. O crescente reconhecimento do papel da ciência em relação à tecnologia e à produção tornou-se uma prioridade nacional para fortalecer esse vínculo" (LUNDVALL, 1992, p. 23, tradução nossa).

Além de ser utilizado como conceito analítico, que identifica as redes de colaboração entre as instituições dos setores públicos e privados envolvidos com a geração e difusão de inovações, o SNI também pode atuar como instrumento de política para forjar e promover essas relaçóes.

O Modelo da Tripla Hélice, formulado por Etzkowitz e Leydesdorff (1995; 2000), também pode ser considerado uma alternativa à abordagem restrita e na literatura é tido como um modelo que evoluiu a partir do SNI. Na contramão dos métodos lineares, nos quais prevalece a ideia de que a inovação está associada às empresas somente, o Triple Helix é representado por uma espiral com três hélices que se entrelaçam por meio de múltiplas interações entre os três âmbitos por eles 
representados: a universidade, a indústria e o governo (CONDE; ARAÚJO-JORGE, 2003). A inovação surge como fruto das interações e do ambiente criado pela rede de colaboração entre os três agentes. Neste modelo, as universidades são consideradas agentes de pesquisa básica, dando suporte ao governo e/ou à indústria, mas também são consideradas empreendedoras, pois, podem comercializar tecnologias, por exemplo. As universidades podem desempenhar um papel significativo no desenvolvimento da inovação em sociedades que têm suas bases cada vez mais sustentadas no conhecimento (ETZKOWITZ; LEYDESDORFF, 1995; 2000).

A Suécia é um exemplo, citado por Edquist (1997), de economia que optou por um modelo de desenvolvimento centrado no formato Tripla Hélice. Ao perceber a perda de competitividade de sua indústria de commodities, resolveu reestruturá-la em meados dos anos 90. Essa mudança estrutural foi realizada a partir da organização de processos cooperativos entre produtores de commodities, produtores de bens de capital para esse segmento, centros públicos de pesquisa e empresas locais de software, sob a coordenação conjunta do governo sueco e da confederação da indústria. O resultado líquido foi a mudança do padrão de especialização e a maior agregação de valor no país. No plano institucional, o governo sueco promoveu uma importante mudança, no início da primeira década de 2000. E o ponto mais significativo dessa mudança foi a criação de agências de inovação, sendo a principal entre elas a VINNOVA, para focalizar as ações de política (EDQUIST, 2003).

O modelo de Tripla Hélice tem se destacado, pois, gera uma estrutura de conhecimento que sobrepõe às esferas institucionais, ou seja, o conhecimento não fica circunscrito somente à empresa ou ao Estado, mas tem um efeito de transbordamento, que faz com que as áreas representadas nas hélices assumam cada vez mais papeis distintos e organizações híbridas emerjam nas interfaces destes processos.

\section{POLÍTICA DE INOVAÇÃO: UM PORQUÊ}

Quando pensamos no fomento da inovação na economia, a presença do Estado é algo que não podemos dissociar. Durante o auge do neoliberalismo, os Estados jamais deixaram de intervir para fomentar o desenvolvimento produtivo e tecnológico e a expansão de setores estratégicos para a dinâmica estrutural, mesmo que estas políticas fossem camufladas por imperativos estratégico-militares (ERBER; CASSIOLATO 1995). Mazzucato (2015) traz à tona diversos exemplos em que o Estado foi, senão o único, um dos maiores responsáveis pelo desenvolvimento de inovaçôes na economia. 
A presença de políticas de inovação na economia de um país permite não só o surgimento de novas ideias e oportunidades de criação de produtos e mercados, mas também o desenvolvimento e difusão de novas tecnologias por meio da promoção das atividades de P\&D e do estímulo à difusão e cooperação de longo prazo nas áreas de pesquisa, investimento estatal e privado. Explicitamente, também visam a promover a consolidação das bases regionais para o desenvolvimento tecnológico, o reforço de malhas de pequenas e médias empresas e o desenvolvimento de atividades consideradas estratégicas para o crescimento econômico doméstico. As políticas de inovação, quando implantadas, servem de instrumentos de estímulo à formação de novas instituições e organizações que visam a interação dos demais atores (universidade, empresas) na busca pela inovação em diversos setores da economia (ERBER; CASSIOLATO, 1995).

Além dos projetos de pesquisa e desenvolvimento conjuntos, as políticas de inovação têm impactos além das fronteiras econômicas, a saber, em áreas como o meio ambiente e a sociedade de uma forma geral (novos ambientes de trabalho, igualdade de gênero, desenvolvimento social). Ressalta-se que estas políticas voltadas para a promoção da interatividade de forma alguma substituem as ações de apoio público à infraestrutura científica e tecnológica (CASSIOLATO; LASTRES, 2005).

Carlota Pérez (2012) descreve que, no passado, as políticas de inovação sempre foram entendidas como uma adição marginal às políticas de desenvolvimento, muito mais ligadas à pesquisa científica e à educação do que ao crescimento econômico, ao emprego e ao bem-estar social. Atualmente, tal cenário foi alterado e as políticas de inovação tornaram-se um elemento central nos esforços destinados a alcançar (catching up) e avançar no crescimento e desenvolvimento econômico não apenas nos países ricos, mas também nos emergentes. Pérez (2012) reforça, no entanto, que a compreensão de tais políticas de inovação no processo de desenvolvimento e crescimento econômico só é possível através (do entendimento) da interação entre a economia, tecnologia e ciência e instituições.

\section{POLÍTICA DE INOVAÇÃO: QUAL POLÍTICA?}

De acordo com Banco Mundial (2010), a existência de políticas que fomentem a inovação e permitam o acesso ao conhecimento é fundamental. As políticas de inovação devem ter em conta as especificidades de cada país em ordem de atender as necessidades locais. A ideia de "one size fits all" é atualmente amplamente 
rejeitada porque há a necessidade de se entender as motivaçôes e os comportamentos específicos que levam as pessoas a inovar. Nessa perspectiva, cada país representa um caso específico com atores e instituições específicos e com relações únicas entre eles. Segundo Fagerberg (2014), não existem soluções políticas universais ou instrumentos que possam ser efetivamente implementados independentemente do contexto concreto do país:

Como resultado, os sistemas nacionais de inovação podem diferir grandemente, (...) e uma combinação de políticas que funcionam em um país pode ser totalmente inadequada em outro. Por conseguinte, a adoção de uma abordagem baseada no sistema de inovação conduz a uma atitude cética em relação ao aconselhamento em matéria de políticas que defende a mesma solução em todas as regiōes, independentemente das diferenças contextuais. (FAGERBERG, 2014, p. 9, tradução nossa)

Além disso, os aspectos culturais que impactam tais decisões hoje são reconhecidos não apenas como variáveis entre países, mas muitas vezes, são variáveis dentro de um próprio país, entre seus estados, cidades ou pequenas organizações sociais.

Assim, na decisão da formulação de uma política de inovação para países em desenvolvimento, vários pontos precisam ser enfatizados: 1) estratégia tecnológica deve se aproveitar o conhecimento e tecnologia global na promoção da economia local e o país deve ter como prioridade para o fomento da inovação a utilização e o desenvolvimento de conhecimentos que já existam, o que será menos oneroso e menos arriscado para suas economias; 2) questôes institucionais - deve se assegurar que muito além de burocracias, os países possuam instituições que suportem projetos inovadores, seja através de provisão técnica ou financeira, para desenvolver setores que, muitas vezes, são precários nos países emergentes, tais como; saúde, educação e meio ambiente; 3) agentes de mudança - possuir atores ou instituições que se utilizem de conexões locais (fontes externas) para alavancar mudanças domésticas; 4) possíveis reformas - atuar em áreas específicas de forma a estimular mudanças amplas (broad approach) e 5) características culturais e comportamentais - respeitar os limites culturais e locais do país.

Serão as políticas voltadas para estes aspectos que farão a ponte entre as instituiçôes, empresas e universidades e o conhecimento já disponível.

Porém, vale lembrar que o surgimento da inovação não depende somente das políticas de inovação. É necessário que, por trás de cada política, haja instrumentos fortes e bem desenhados que possam suportar e nutrir de forma sustentável as diversas áreas das políticas de inovação em países com economia emergente. 


\section{CONSIDERAÇÕES FINAIS}

Embora a noção consistente do que é política de inovação seja recente, ela tem se mostrado cada vez mais componente essencial na formulação das políticas públicas. $\mathrm{O}$ interesse crescente dos formuladores de políticas públicas com o processo de inovação reflete a importância que a inovação tem nas mudanças sociais e econômicas de um país.

As políticas de inovação tornaram-se componente central dos esforços para alcançar (catching up) e acelerar o desenvolvimento econômico não só nos países ricos, mas principalmente nos emergentes. A contribuição da política de inovação para o processo de desenvolvimento econômico depende de como tal política incorpora (ou considera) as interaçooses entre economia, tecnologia, ciência e instituições. Deve-se ter uma abordagem ampla que considere todas as políticas e seus instrumentos, bem como os agentes envolvidos, que intervém no processo de inovação e na formação do ambiente no qual se dá a inovação.

A formulação de políticas de inovação de forma eficaz é uma tarefa exigente, que requer uma compreensão profunda do contexto em que estará inserida. Os sistemas nacionais de inovação podem diferir significativamente. Uma combinação de políticas que funcione em um país pode não funcionar em outro. É necessário respeitar os limites locais (o que inclui os culturais) e estimular a inovação tendo como base o conhecimento já existente, o que seria menos oneroso e arriscado. Deve-se também priorizar setores que muitas vezes são precários nos países emergentes como saúde, educação e meio ambiente. Além disso, é importante apoiar a formação de policymakers e boas organizações de suporte à inovação. Os países devem ter burocracias eficientes que suportem projetos inovadores, seja por meio da provisão técnica ou financeira. Os países emergentes devem promover as interaçôes locais dos agentes envolvidos na criação e difusão da inovação (empresas, universidades e governos) e simultaneamente garantir as trocas de conhecimento e experiência com agentes de outros países. A internacionalização do processo de inovação é um fenômeno atual e marcante em diversos países bem-sucedidos.

\section{REFERÊNCIAS}

BORRÁS, S; EDQUIST, C. The Choice of Innovation Policy Instruments. Circle - Lund University. Sweden, 2013. 
CASSIOLATO, J; LASTRES, H. Innovation Systems and Development., in International Development: Ideas, Experience, andProspects. Disponível em: <http://www.globelicsacademy. org/2013_pdf/Readings/Cassiolato\%20at\%20all\%20_2.pdf. Acesso em: 15 mar. 2017.

CONDE, V. F, MARIZA.; ARAÚJO-JORGE, T. Modelos e concepções de inovação: a transição de paradigmas, a reforma da C\&T brasileira e as concepçóes de gestores de uma instituição pública de pesquisa em saúde. Disponível em: <http://www.scielo.br/scielo.php ?pid=S1413-81232003000300007>. Acesso em: 25 abr. 2017.

ERBER, F, CASSIOLATO, J. Política Industrial: teoria e prática no Brasil e na OCDE. Disponível em: <http://www.rep. org.br/PDF/66-3.PDF>. Acesso em: 28 mar. 2017.

EDQUIST, C; HOMMEN, L; TSIPOURI, T. Public Technology Procurement and Innovation. New York: Springer US, 1997.

ETZKOWITZ, H; LEYDESDORFF, L. The Triple Helix---University Industry-Government Relations: A Laboratory for Knowledge-Based Economic Development. Disponível em: <https:// www.researchgate.net/publication/241858820>. Acesso em: 12 abr. 2017.

. The dynamics of innovation: from National Systems and "Mode 2" to a Triple Helix of university-industry-government relations. Disponível em: http://www.chss.uqam.ca/ Portals/0/docs/sts8020/(20)Etzk-Leides.Triple.Helix.pdf. Acesso em: 03 mar. 2017.

FAGERBERG, J. Innovation: A Guide to Literature. Centre for Technology, Innovation and Culture. Oslo: University of Oslo, 2003.

Innovation policy: in search of a useful theoretical framework. Science and innovation policy: Dynamics, Challenges, Responsibility and Practice, jun. 2014, UK.

. Innovation policy, national innovation systems and economic performance: in search of a "useful" theorical framework. Disponível em: <http://www.janfagerberg.org/wpcontent/uploads/2015/10/2015-Atlanta-Fagerberg-innovation-policy-1.pdf>. Acesso em: 17 mar. 2017.

FAGERBERG, J; MOWERY, D; NELSON, R. The Oxford Handbook of Innovation. Oxford: Oxford University Press, 2006.

FOXON, T; MAKUCH, Z; MATA, M. \& PEARSON, P. Innovation Systems and Policy Making for the Transition to Sustainability. In: KLAUS, J, MANFRED, B. WIEEZOREK, A (Ed). Governance for Industrial Transformation. Berlim: Environmental Policy Research Centre, 2004.

FREEMAN, C.; SOETE, L. A economia da inovação industrial. Campinas: Editora da Unicamp, 2008.

INNOVATION POLICY PLATFORM - IP. OECD, World Bank. Disponível em: <http://www.innovationpolicyplatform.org. Acesso em: 09 fev. 2017.

LUNDVALL, B.-A, Innovation System Research and Policy Where It Came From and Where It Might Go. Disponível em: <http://www.globelicsacademy.org/2011_pdf/Lundvall_ (post\%20scriptum).pdf>. Acesso em: 29 mar. 2017. 
National Systems of innovation: Towards a theory of innovation and interactive learning. Londres: Pinter, 1988; 1992.

MAKÓ, C; ILLÉSSY, M. Innovation Policy Review - National and European Experience. Mai. 2015. (QuInnE Working Paper, 1).

MAZZUCATO, M. O Estado Empreendedor. São Paulo: Penguin, 2014.

OECD. Innovation Strategy: Getting a Head Start on Tomorrow, Paris, 2005.

OECD. Innovation for Development. Paris, 2012. Disponível em: <http://www.oecd.org/ innovation/inno/50586251.pdf>. Acesso em: 16 mar. 2017.

OECD. Innovation for Development. Paris, 2014. Disponível em: <http://www.oecd.org/sti/ inno/knowledge-and-innovation-for-inclusive-development.htm>. Acesso em: 16 mar. 2017.

PÉREZ, Carlota. Innovation systems and policy for development in a changing world. In: E, S, Andersen; J. Fagerberg e B. Martin (org.) Innovation Studies: Evolution and Future Challenges. Oxford: Oxford University Press, 2013.

SCHIENSTOCK, G; HAMALAINEM, T. Transformation of the Finnish Innovation System - A Network Approach. Disponível em: <https://pdfs.semanticscholar.org/bf83/11 67c5aae0f5c4042661575145272386c2bc.pdf>. Acesso em: 24 mar. 2017.

WORLD BANK. Innovation Policy - A Guide for Development Countries. Disponível em: <https://openknowledge.worldbank.org/bitstream/handle/10986/2460/548930PUB0E PI11C10Dislosed061312010.pdf>. Acesso em: 24 mar. 2017. 
